\title{
Characterization of Traumatic Brain Injury Research in the Middle East and North Africa Region: A Systematic Review
}

\author{
Samar Al-Hajj ${ }^{a}$ Zeinab Hammoud ${ }^{a} \quad$ Jure Colnaric $^{b} \quad$ Maya Ataya $^{b}$ \\ Marie Michele Macaron ${ }^{b}$ Kamil Kadi $^{b}$ Hayat Haratic Helen Phipps ${ }^{d}$ \\ Stefania Mondello ${ }^{e}$ Hani Tamim $^{f}$ Hussein Abou Abbass $^{\text {b, } c, g}$ Firas Kobeissy $^{\text {b, }}$ h
}

the TBI Working Group

\begin{abstract}
${ }^{a}$ Health Management and Policy Department, Faculty of Health Sciences, American University of Beirut, Beirut, Lebanon; bepartment of Biochemistry and Molecular Genetics, Faculty of Medicine, American University of Beirut, Beirut, Lebanon; 'Neuroscience Research Center, Faculty of Medicine, Lebanese University, Beirut, Lebanon; ${ }^{d}$ Booz Allen Hamilton, San Antonio, TX, USA; 'Department of Biomedical and Dental Sciences and Morphofunctional Imaging, University of Messina, Messina, Italy; fDepartment of Internal Medicine, American University of Beirut Medical Center, Beirut, Lebanon; 9Department of Surgery, Makassed General Hospital, Beirut, Lebanon; hProgram for Neurotrauma, Neuroproteomics and Biomarkers Research, Departments of Emergency Medicine, Psychiatry, Neuroscience and Chemistry, University of Florida, Gainesville, FL, USA
\end{abstract}

\section{Keywords}

Traumatic brain injury · Mild traumatic brain Injury · CT scan · Middle East and North Africa region · Epidemiology

\begin{abstract}
Objectives: Traumatic brain injury (TBI) represents a major health concern worldwide with a large impact in the Middle East and North Africa (MENA) region as a consequence of protracted wars and conflicts that adversely affect the general population. Currently, systematic TBI studies in the MENA region are lacking, nonetheless they are immensely needed to enhance trauma management and increase survival rates among TBI patients. This systematic review aims to characterize TBI in the MENA region to guide future policy choices and research efforts and inform tailored guidelines capable of improving TBI management and patient treatment and outcome. Furthermore, it will serve as a road map to evaluate and assess knowledge of trauma impact on
\end{abstract}

\section{S.A.-H. and Z.H. contributed equally.}

$\begin{aligned} & \text { karger@karger.com } \\ & \text { www.karger.com/ned }\end{aligned}$
Karger $\%$

regional health systems that can be adopted by health-care providers to raise awareness and improve trauma care. Methods: We conducted a comprehensive search strategy of several databases including MEDLINE/Ovid, PubMed, Embase, Scopus, CINAHL, Google Scholar, and the grey literature in accordance with the PROSPERO systematic review protocol CRD42017058952. Abstracts were screened, and selected eligible studies were reviewed independently by 2 reviewers. We collected demographics information along with TBI characteristics, mortality rates, and regional distribution. Data were extracted using REDCap and checked for accuracy. Results: The search strategy yielded 23,385 citations; 147 studies met the eligibility criteria and were included in this review. Motor vehicle accident (MVA) was the lead-

Firas Kobeissy

Department of Biochemistry and Molecular Genetics, Faculty of Medicine American University of Beirut, Riad El-Solh 1107-2020, PO Box 110236 Beirut 32608 (Lebanon)

firasko@gmail.com

Samar Al-Hajj

Faculty of Health Sciences, American University of Beirut

Riad El-Solh 1107-2020, PO Box 110236

Beirut 32608 (Lebanon)

sh137@aub.edu.lb

Hussein Abou Abbass

Department of Biochemistry and Molecular Genetics, Faculty of Medicine American University of Beirut, Riad El-Solh 1107-2020

PO Box 110236, Beirut 32608 (Lebanon)

abouabbass.hussein@gmail.com 
ing cause of TBI ( $41 \%)$ in the MENA region, followed by the military- (15.6\%) and fall- (8.8\%) related TBI. Males predominantly suffer from TBI-related injuries (85\%), with a high prevalence of MVA- and military-related TBI injuries. The TBI mortality rate was $12.9 \%$. The leading causes of mortality were MVA (68\%), military (20.5\%), and assault (2.9\%). The vast majority of reported TBI severity was mild $(63.1 \%)$ compared to moderate (10.7\%) and severe TBI (20.2\%). Patients mainly underwent a Glasgow Coma Scale assessment (22.1\%), followed by computed tomography scan (8.9\%) and surgery (4.1\%). Conclusions: Despite its clinical, social, and economic burden, the evidence of TBI research in the MENA region is scarce. Further research and high-quality epidemiological studies are urgently needed to gain a deep understanding of the TBI burden in the region, facilitate the allocation of adequate resources, implement effective preventive and intervention strategies and advise on the TBI patient management as reflective on the TBI patterns and modes.

(c) 2021 S. Karger AG, Basel

\section{Introduction}

Traumatic brain injury (TBI) represents one of the leading causes of death and long-term disability globally $[1,2]$. An estimated 69 million (95\% UI 64-74 million) individuals sustained a form of TBI in 2017, resulting in emergency visits and hospitalizations [3]. TBI is defined as an acute injury to the brain caused by a penetrating head injury or by an external physical force to the head/ face (i.e., a blow, a bump, or a jolt), leading to the impairment of neurological functions, and in many cases, the disruption of physical, cognitive, and mental functioning [1]. Depending on the severity of the TBI (i.e., mild, moderate, or severe), a range of symptoms manifest with a particular impairment associated with each TBI severity level. These symptoms span across a wide spectrum, ranging from physical impairment, including loss of consciousness (LOC), dizziness, and blurred vision, to behavioral and mood changes.

Low- and middle-income countries bear a large burden of TBI, with an estimated number of cases 3 times larger compared to high-income countries [4]. An examination of the external causes of TBI in the Middle East and North Africa (MENA) region shows high rates of TBI associated with war operations, conflicts and violence, road traffic injuries, and fall injuries [5]. These reportedly high rates stem from protracted regional wars, constant political instability coupled with major defi- ciencies in government enforcement of safety regulations, which exacerbate the TBI problem in the region. According to the Global Burden of Disease 2017, more than 1.8 million individuals suffered from TBI in 2017 in the MENA region [3]. The incidence rate for minor and moderate/severe TBI in 2017 was 176.8 and 134.9 per 100,000 population, respectively. Despite its substantial human burden, a limited number of studies investigated the epidemiology of TBI in the MENA region. Existing regional studies were limited in their scopes and systematic approach, focusing mainly on a particular type of TBI and its population, or geographic area, which limits data use, potential to draw conclusions, enhance patient management, increase survival rates and decrease mortality as discussed by Hejase et al. [6]. To the best of our knowledge, there has not been any systematic review exploring TBI and its epidemiology in the MENA region.

Here, we aim to conduct a systematic review of the literature to investigate characteristics and epidemiological regional patterns of TBI in the MENA, analyzing TBI types, external causes, clinical presentation, and disposition. This review will help to understand the breadth and depth of the existing TBI literature in the region and to develop benchmarks for evaluating existing TBI quality of care. Furthermore, such information will be a vital step towards identifying best clinical practices, appropriately allocating resources, and setting critical research priority areas. Evidence from this review will contribute to informing policy makers and supporting the development of strategic tailored guidelines for improving the management and treatment of TBI in the MENA region.

\section{Methodology}

Study Registration and Search Strategy

The systematic review was conducted according to our registered protocol (PROSPERO CRD42017058952; available on: https://www.crd.york.ac.uk/prospero/display_record. php?ID=CRD42017058952). The design is consistent with the recommendations made in the "Cochrane Handbook for Systematic Reviews and Meta-Analysis," with reporting based on the Preferred Reporting Items for Systematic Reviews and Meta-Analyses (PRISMA) guidelines [7]. We searched the following electronic databases: MEDLINE/Ovid, PubMed, Embase, Scopus, CINAHL, and Google Scholar (see online suppl. material; for all online suppl. material, see www.karger.com/doi/10.1159/000511554) from their inception till August 2019. Screening was restricted to languages English, French, and Arabic. Related key index and $\mathrm{MeSH}$ terms were selected in the search strategy, including "Brain injury" and "Brain trauma" or "Brain concussion," "Brain hemorrhage," "Brain bleeding," "Middle Eastern," "North Africa" (see online suppl. material for a full search strategy). Grey literature was 
Fig. 1. PRISMA chart design for TBIs in the MENA region. The PRISMA chart was designed to quantify the prevalence and epidemiology of TBIs in the MENA region. TBI, traumatic brain injury; MENA, Middle East and North Africa; PRISMA, Preferred Reporting Items for Systematic Reviews and Meta-Analyses.

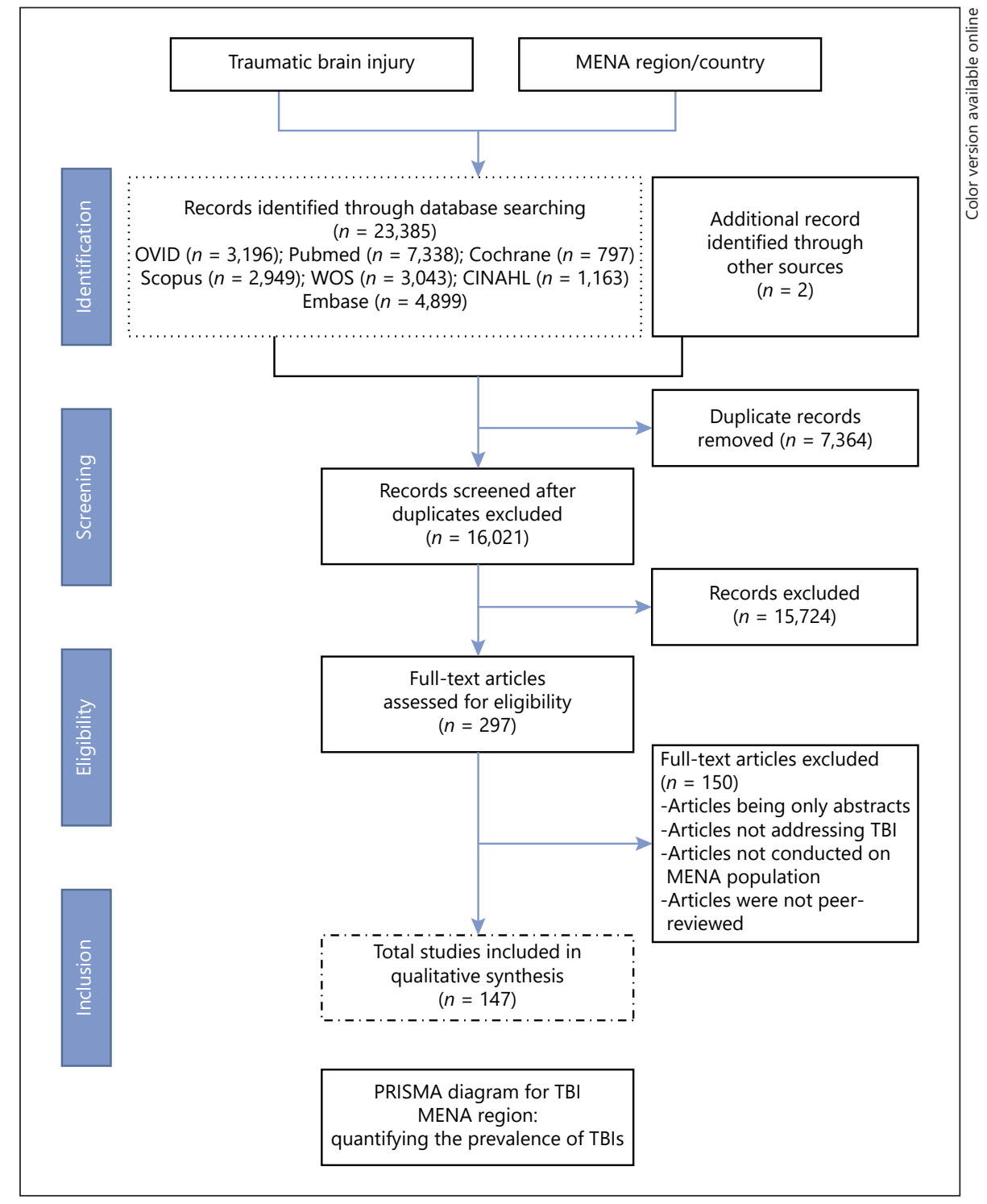

searched, including conference proceedings, dissertations, governmental records, and non-government organizations' documents. Cited and citing reference lists of all included studies were also examined for relevant articles.

\section{Inclusion Exclusion Criteria}

Retrieved studies were eligible for inclusion if they (1) investigated patients (outpatient or inpatient) with an admission diagnosis of TBI that is confirmed by a physician and (2) were unpublished or published cross-sectional, case-control, prospective, retrospective, and clinical trial studies in the MENA region. The MENA region refers to the following countries: Algeria, Bahrain, Afghanistan, Djibouti, Egypt, Iraq, Jordan, Kuwait, Lebanon, Libya, Malta, Morocco, Oman, Qatar, Saudi Arabia, Syria, Tunisia, United Arab Emirates, Palestine (West Bank and Gaza), and Yemen. Iran, Turkey, and Israel were not included in this review. Articles were excluded if the article was only abstract, conference lecture, non-TBI, in a language other than English, French, or Arabic and if the reported TBI study was conducted outside the MENA region as defined above.

\section{Article Screening}

Article titles and abstracts were screened by at least 2 independent investigators (Z.H., J.C., M.A., K.K., and H.A.) after removing duplicates using EndNote. The reviewers (Z.H., J.C., M.A., K.K., and H.A.) retrieved the full texts of articles that met the study's eligibility criteria. Kappa statistics were calculated to assess and evaluate the agreement between the 2 reviewers who screened the articles for eligibility. The REDCap software was adopted to design the data collection form and to capture variables. Two other reviewers (Z.H. and M.A.) - independently and in duplicate screened the full texts of the articles identified by the first 2 reviewers and completed the database. In cases of disagreement, senior reviewers (S.A. and F. K.) were consulted to resolve the disparity. 
Table 1. Regional distribution of TBI studies, sample size, and severity (mild, moderate, and severe) across counties in the MENA region

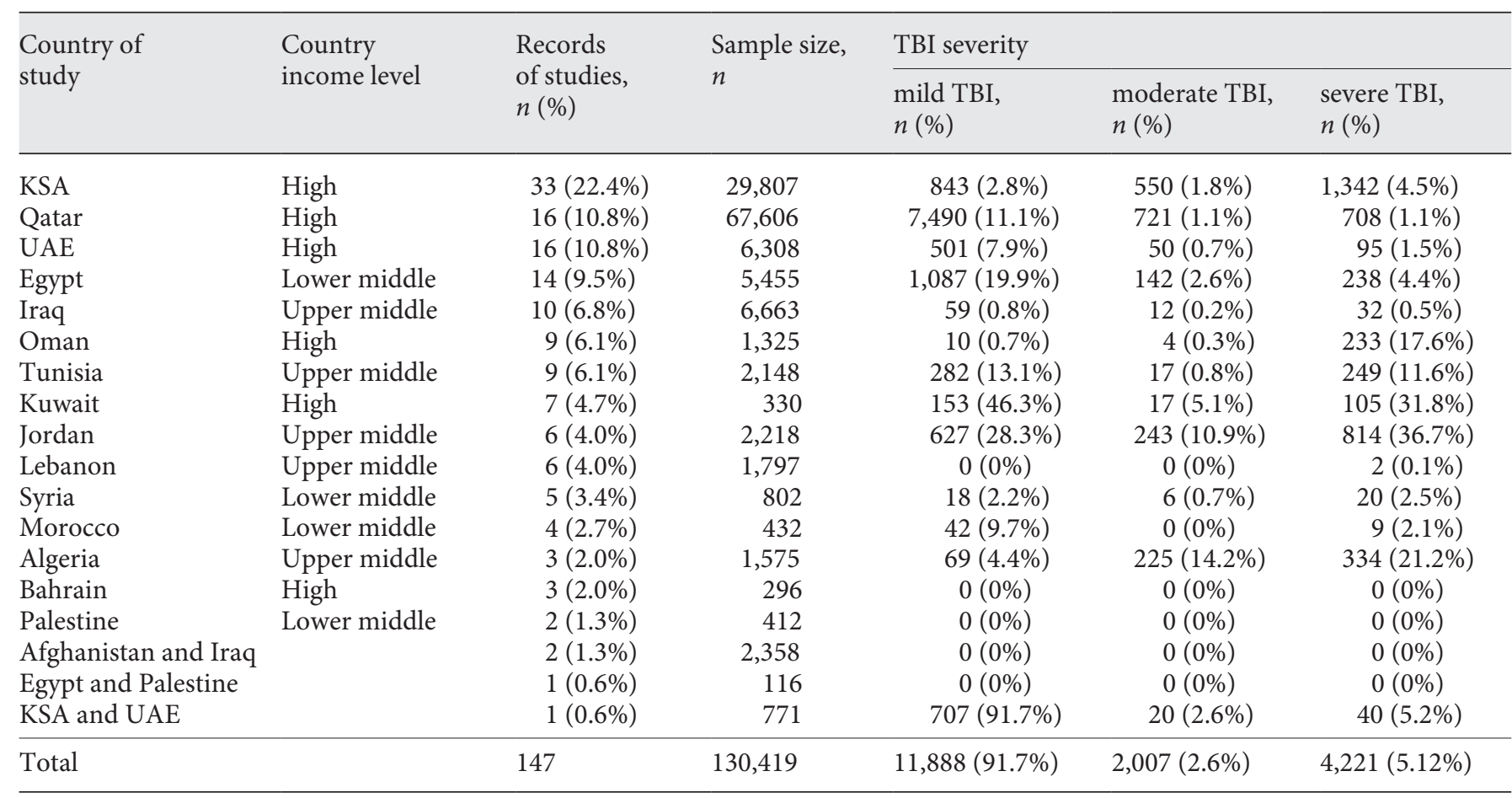

KSA, Kingdom of Saudi Arabia; TBI, traumatic brain injury; MENA, Middle East and North Africa.

Data Abstraction and Analysis

The Methodological Evaluation of Observational Research (MORE) checklist was adopted as a framework to assess the bias and the quality of the eligible studies. All studies meeting the eligibility criteria were retrieved. Study details abstracted from each article onto the REDCap form, including title, author, publication year, study design, study time period, source of population, country, site of study, sample size, case ascertainment method, gender, age groups, severity (documented severity, Glasgow Coma Scale [GCS]), LOC, duration of LOC, Injury Severity Score (ISS), Abbreviated Injury Scale (AIS), computed tomography (CT) findings, cause of injury, mechanism of injury, and outcome measures reported (i.e., incidence, prevalence, mortality, and case-fatality). Descriptive analysis of the retained studies was conducted to assess the distribution of TBI literature across the MENA region, the gender distribution, and the mortality and severity rate of sustained TBIs.

\section{Results}

The search strategy yielded 23,385 articles. After removing duplicates and conducting the initial screening, only 297 met the study criteria and were included for a full-text review. Of these, 150 were excluded for multiples reasons including articles being only abstracts, not ad- dressing TBI, were not conducted on the MENA population, and were not peer-reviewed articles. A total of 147 full-text articles and reports were included in the final review (please refer to online suppl. data 1 for details on included studies) (refer to PRISMA chart Fig. 1).

\section{TBI Study Characteristics}

The included studies reported cases of TBI within a selected sample frame, mainly the targeted population, such as patients sustaining a TBI and presenting to regional emergency departments or patients with TBI admitted to hospitals. TBI studies were mainly investigating patients' records retrieved from hospitals and medical centers, rehabilitation facilities, trauma center, and military combat support facilities. TBI mortality and severity levels were the most commonly used measures for TBI assessment.

\section{TBI Regional Distribution}

The studies were distributed across the MENA region with the Kingdom of Saudi Arabia (KSA) reporting the highest number of TBI publications (22\%), followed by Qatar and UAE (10.8\%), Egypt (9.5\%), Iraq (6.8), and Oman and Tunisia (6.1\%). The remaining MENA coun- 


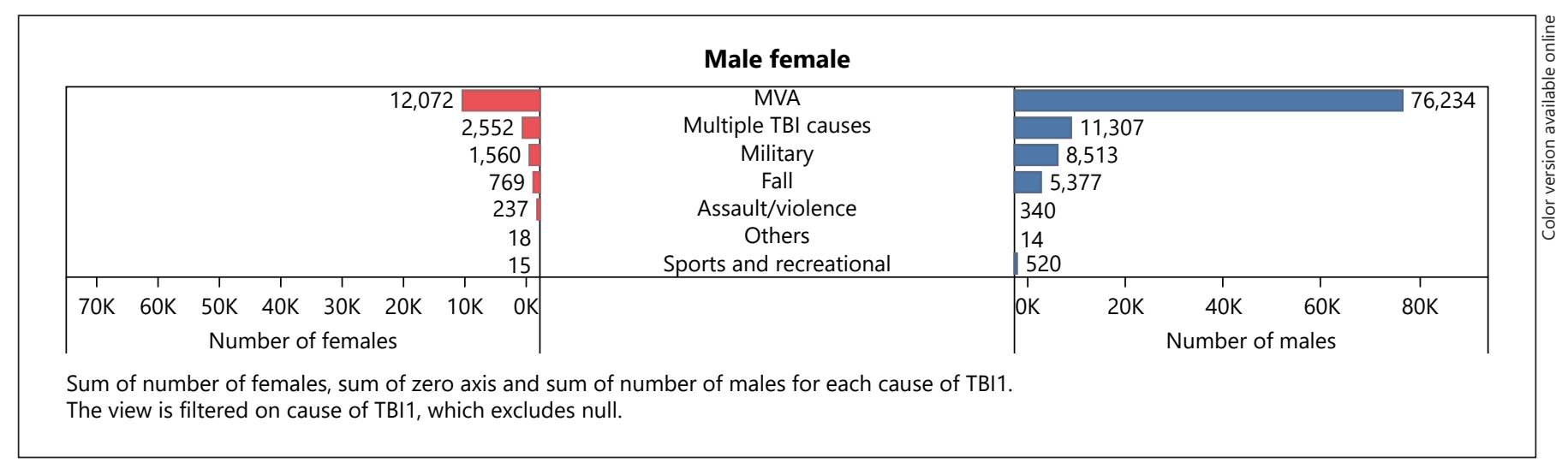

Fig. 2. Male to female distribution across TBI incidence. This figure shows the sum of the number of males and females for each cause of TBI in a descending order from the TBI mechanism with the highest number of males and females to the lowest number. MVA, motor vehicle accident; TBI, traumatic brain injury.

tries reported less than 10 studies. One study reported data from 2 countries: KSA and UAE (Table 1).

\section{TBI Gender Distribution}

This systematic review found that males predominantly suffered from TBI-related injuries for all mechanisms of TBI, with an M:F ratio of 5.9. The total number of males with TBI was 102,305 cases, corresponding to a proportion of $85 \%$ of the review-accumulated sample size, compared to 17,223 cases for females (15\%) (see Fig. 2). The most common TBI mechanism sustained by males were MVAs. Evident gender differences were illustrated across all TBI mechanisms. A remarkably high prevalence of male TBI victims was reported for MVAs, military-, and fall-related TBI with M:F ratio of 6.3, 5.4, and 6.9, respectively. Other TBI mechanisms including assault/violence reported approximately equal prevalence for both males and females (Table 2). Nevertheless, existing studies hindered the possibility of assessing gender differences among age groups due to the incomplete and non-standardized documentation.

\section{TBI Mechanism or External Causes}

TBIs resulted from several injury mechanisms (Fig. 3). Fig. 3 represents a TreeMap of the TBI landscape in the MENA region.

\section{Motor Vehicle Accidents}

Motor vehicle accident (MVA) was the most prevalent mechanism of TBI-related injuries in this review. More than $40 \%(N=61)$ of the included studies solely reported MVA-related TBI. MVA incidents represented a substan-
Table 2. Gender distribution of TBI studies in the MENA region

\begin{tabular}{lrrr}
\hline Cause of TBI & $\begin{array}{l}\text { Number } \\
\text { of females }\end{array}$ & $\begin{array}{l}\text { Number } \\
\text { of males }\end{array}$ & $\begin{array}{l}\text { Ratio } \\
\text { M:F }\end{array}$ \\
\hline MVA & 12,072 & 76,234 & 6.3 \\
Multiple TBI causes & 2,552 & 11,307 & 4.4 \\
Military & 1,560 & 8,513 & 5.4 \\
Fall & 769 & 5,377 & 6.9 \\
Assault/violence & 237 & 340 & 1.4 \\
Others & 18 & 14 & 0.7 \\
Sports and recreational & 15 & 520 & 34.66 \\
\hline Total & 17,223 & 102,305 & 5.9 \\
\hline
\end{tabular}

MENA, Middle East and North Africa; MVA, motor vehicle accident; TBI, traumatic brain injury; M, male; F, female.

tial health problem that overburdened all the MENA countries without any exception and was evident in the overwhelming number of reported studies in the MENA countries, documenting the severity of sustained head trauma across all age groups and all populations including children and youth MVA [8-10], the elderly [11], and the adult populations [12-17]. Of interest, MVA-TBI studies were not limited to vehicle-related injuries; they included all types of road users, namely pedestrians [18, 19], motorcyclists [20], bicyclists [21], and all-terrain vehicles (ATVs) riders [22].

\section{Military}

Twenty-three studies reported military-related TBI, mainly occurring in countries with lengthy civil wars,
Al-Hajj et al. 
Fig. 3. TreeMap visualization of the landscape of TBI literature. This Tableau TreeMap visualization depicts the landscape of TBI literature in the MENA region by cause of TBI, the sum of number of records, and the percentage of total number of records for each TBI cause. Color indicates the cumulative number of studies (red the highest and green the lowest). The portion size reflects the total number of studies. MENA, Middle East and North Africa; MVA, motor vehicle accident; TBI, traumatic brain injury.

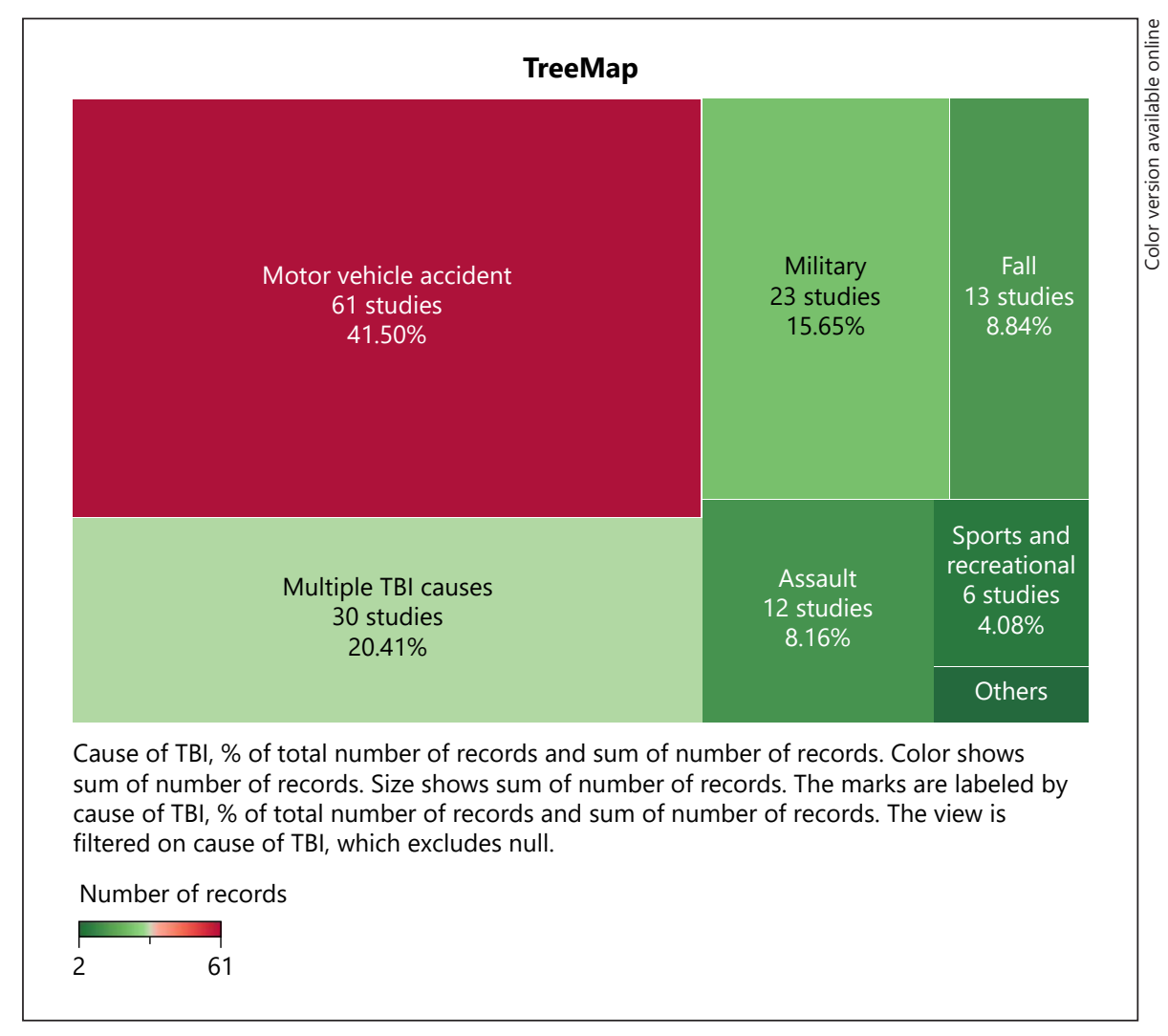

conflicts, and political unrest, including Syria [23-25], Lebanon [26-30], Iraq [31, 32], Kuwait [33, 34], and Afghanistan $[17,32]$. The vast majority of military TBI victims were males $(81 \%)$. It is worth noting that although women sustained less severe cases of TBI, they suffered more severe outcomes. Causes of military-related TBI included wounds triggered by blast injuries from high-velocity explosives and cluster bombs (41.3\%), gunshot (21.1\%), fragmentation (16.5\%), land mines and general missiles injuries (19.2\%), as well as wounds from improvised explosive devices. Five TBI military injury studies focused on the pediatric population, documenting children's injury characteristics and outcomes $[25,31,32$, 35]. Procedures for management and treatment of TBI patients ranged from simple debridement, craniotomy, emergency surgery, and bacterial treatment to more complex surgical interference, including intracranial hematoma evacuation and bullet extraction as well as reconstructive craniofacial surgery.

\section{Falls}

Fall-related TBI was the third leading cause of TBI injuries $(N=13)$ in the MENA region. Accidental fall from height was the primary cause of fall-related TBI reported in the literature; it included different heights ranging from high altitudes (i.e., buildings) to ladders, stairs, and beds. These reported TBIs were most common among the pediatric and the elderly population, occurring mainly at schools and homes [36-38]. Fall from height was a major injury mechanism sustained by male expatriate workers and primarily occurring as a result of falling from heights at construction sites or being struck by falling objects [39, 40]. One study reported fall injuries due to military parachuting causing hemorrhage and resulting in fatal injuries [41]. The only incidence rate presented within these fall articles was 206 per 100,000 population per year.

\section{Assault/Violence}

Twelve studies reported non-military violence-/assaults-related TBI. Most of these studies portrayed assault as an infrequent mechanism of TBI compared to other more prevalent causes (e.g., MVAs and military). Nine out the 12 studies identified cases of child abuse and described victims' characteristics and TBI clinical presentations, namely, intracranial and skull fractures, skeletal injuries, scalp swelling, and subgaleal hematoma [42-46]. The majority of abused children were below the age of 6 with the exception of one study that documented fatal 
Table 3. TBI outcomes (mortality: mild, moderate, and severe TBI) in the MENA region

\begin{tabular}{lccrr}
\hline Cause of TBI & $\begin{array}{l}\text { Number of } \\
\text { patients with mild TBI }\end{array}$ & $\begin{array}{l}\text { Number of patients } \\
\text { with moderate TBI }\end{array}$ & $\begin{array}{l}\text { Number of } \\
\text { patients with severe TBI }\end{array}$ & $\begin{array}{l}\text { In-hospital } \\
\text { mortality }\end{array}$ \\
\hline MVA & 10,107 & 1,746 & 3,764 & 3,179 \\
Fall & 1,655 & 213 & 490 & 26 \\
Multiple TBI causes & 36 & 20 & 253 & 335 \\
Sports and recreational & 29 & 19 & 37 & 13 \\
Others & 28 & 0 & 51 & 4 \\
Military & 18 & 3 & 12 & 957 \\
Assault/violence & 15 & 2,007 & 4,608 & 4,651 \\
\hline Total & 11,888 & & 137 \\
\hline
\end{tabular}

MENA, Middle East and North Africa; MVA, motor vehicle accident; TBI, traumatic brain injury.

head trauma sustained by teenagers, mostly girls (98\%) aged between 12 and 18 [46]. Abusive head trauma (transverse fracture) was the primary injury outcome for abused children. The major reported outcomes of children with severe TBI were vomiting, seizures, LOC, coma, and deaths. Death was a common outcome among victims of inflicted trauma and was documented as death on scene, upon ED arrival or within $24 \mathrm{~h}$ of hospital admission. More than $90 \%$ of the perpetrators are individuals known to the child; most abuses were inflicted by parents and is more common among families with a low socioeconomic status.

One study reported TBI resulting from purposely inflicted torture on prisoners, asylum seekers, and refugees in centers in Gaza and Egypt [47]. One study reported self-inflected nail gun penetrating injury resulting in craniocerebral penetrating trauma [48]. One study reported TBI related to violence resulting in gunshot wounds to the head [49] and identified severe, moderate, and mild TBI (mTBI) present in 40,10 , and $50 \%$ of patients, respectively. Available adult assault data showed that compared to females, males were at higher risk for TBI due to violence/assault. Nevertheless, assault-related TBI resulted in a substantial mortality rate ranging from 17 to $30 \%$ $[42-44,49]$.

\section{Multiple TBI}

Approximately 30 studies investigated multiple types of TBI-related injuries, including MVA, fall, military, and assault [50-65]. The main objectives of these studies were to examine hospital-based epidemiological characteristics of TBI patients, identify the functional and socioprofessional outcome and mortality-predictive factors and in some cases assess the validity of screening instruments including the Functional Independence Measurement (FIM), Disability Rating Scale (DRS), and the Community Integration Questionnaire (CIQ). Moreover, these studies evaluated treatment mode and postoperative clinical and rehabilitation outcomes to inform treatment strategies, clinical practices guidelines, and therapeutic management protocols. Two studies explored multiple TBIs associated with seasonal occasions, such as injuries occuring during the Muslim pilgrimage season [53] and the fasting month of Ramadan [53].

\section{Sports and Recreational}

Sports- and recreation-related TBI were limited in the literature. Four studies reported camel-related injuries in Qatar, UAE, Morocco, and Syria [37, 66-68] and head trauma sustained as a result of camel's kick or fall. The third study reported camel-related fall at touristic sites in Morocco and Palmyra, Syria. One study reported soccer-related TBI injuries [39], exploring incidence, characteristics, and patterns of head injury occurrence at soccer league clubs. Another study reported recreational activities, namely, ATV, jet skiing, soccer, and water boat activities, particularly among the male youth population, resulting in severe head trauma including subarachnoid hemorrhage and subdural and epidural hematoma [17].

\section{Others}

Two studies describing unclassified mechanisms of TBI were also included in this review. One study reported cases of TBI in children resulting from animal encounters caused by donkey bites [69] and the another study documented pediatric TBI caused by falling metallic ceiling fans and resulting in sustained head injuries [70]. 
Table 4. Tools adopted by EMS to measure TBI

\begin{tabular}{lrr}
\hline Tools used & $\begin{array}{l}\text { \% of total } \\
\text { number } \\
\text { of records }\end{array}$ & $\begin{array}{l}\text { Number } \\
\text { records }\end{array}$ \\
\hline GCS & $22.1 \%$ & 32 \\
CT scan & $8.9 \%$ & 13 \\
Surgical & $4.1 \%$ & 6 \\
Multiple tools used (CT, GCS, X-ray, surgical) & $22.7 \%$ & 33 \\
Unspecified & $42.1 \%$ & 61 \\
\hline
\end{tabular}

TBI, traumatic brain injury; GCS, Glasgow Coma Scale; EMS, emergency medical services.

\section{TBI Outcomes}

TBI outcomes were measured in terms of mortality and severity. Table 3 depicts in-hospital mortality and patient distribution according to their level of TBI severity.

\section{TBI Mortality}

Mortality refers to the number of TBI-related deaths within a cohort. TBI fatality case proportion quantifies the number of TBI patients who eventually died as a result of the sustained brain injury in the studies' samples. The proportion of mortality was $4,651 / 35,905=12.9 \%$. The leading cause of TBI mortality was reported among victims of MVAs (68\%) followed by military (20.5\%) and assault (2.9\%).

\section{TBI Severity}

TBI severity, measured by the GCS, is divided into 3 categories: mild, moderate, and severe. There are substantial differences in the proportion of cases reported among TBI severity levels. Out of the 147 compiled studies, the vast majority reported mild (63.1\%) compared to moderate (10.7\%) and severe (20.2\%) TBI.

Severe TBI. Only $20.2 \%(4,608 / 22,708)$ of the included studies reported severe TBI cases. Most of these cases were sustained by MVA victims $(n=974)$ followed by fall- and military-related TBIs. MVAs and falls were major contributors to most TBI cases.

Moderate TBI. The proportion of moderate TBI reported in the included studies was $10.7 \%(2,007 / 18,608)$. Similar to severe TBI, the 2 leading causes of moderate TBI were MVAs and falls.

Mild TBI. The proportion of mTBI reported in review was $63.1 \%(11,888 / 18,838)$. The highest frequency of mTBI was reported among victims of MVAs $(n=10,107)$ followed by falls $(n=1,655)$.

Characterization of Traumatic Brain

Injury in the MENA Region
Tools Adopted

A series of assessment tools was adopted by emergency medical services personnel to assess the severity and consciousness of the TBIs sustained by patients. These tools included the GCS, CT, X-ray, and surgical interventions. The majority of patients reported in the TBI studies underwent a GCS assessment $(22.1 \%)$, followed by CT scan $(8.9 \%)$ and surgery (64.1\%). Almost $22.7 \%$ of TBI patients undertook multiple assessments following their TBIs as shown in Table 4.

\section{Discussion}

TBI imposes a substantial clinical, social, and economic burden on individuals and health-care systems globally. Accurate diagnosis and recognition of cases of TBI across the population are critical to assess the current TBI burden. This comprehensive review is the first to compile MENA studies to investigate TBI epidemiology, mechanisms, gender, regional distribution, severity levels, and management. It offers insights into acknowledging the breadth and depth of the existing TBI literature in the MENA region in an attempt to guide future policy strategies and research efforts and to inform tailored guidelines capable of improving management and treatment of TBI patients, and consequently their outcome. This review will further serve as a road map to evaluate the impact of trauma on regional health systems with the aim to improve trauma care and raise awareness in the general public.

This review reveals that the MENA region bears a disproportionally high prevalence of TBIs compared to North America and Europe, despite the limited TBI literature in the region $[4,71,72]$.

Aligning with international literature, this review showed that males predominantly suffered from TBI-related injuries for all mechanisms of injuries with a proportion of $85 \%$ of the study-accumulated sample size compared to females (15\%). The most common TBI mechanism sustained by the male population was MVA, compared to females who sustained a high proportion of assaults.

Overall, the prevalence of TBI mortality was $12.9 \%$. The leading cause of TBI mortality was reported as MVA (68\%) followed by military-related injuries $(20.5 \%)$ and assault $(2.9 \%)$. This review indicates that high-income MENA countries have a noticeably significant number of MVA-related TBI injuries, reported in $45 \%$ of the KSAincluded studies $(N=15 / 33)$ and $56 \%$ of Qatar $(9 / 16)$ and

Neuroepidemiology 2021;55:20-31 
UAE (9/16) studies. These estimates signal an alarming indicator of the MVA burden across regional MENA countries. This finding is consistent with existing evidence that underscores the high burden of MVA in the region [73], possibly explained by the rapid urbanization of many MENA countries along with major changes in the automobile industry and coupled with lax government-enforced safety regulations that resulted in the increased frequency and severity of MVAs.

This review shows that a high proportion of militaryrelated TBI was reported in MENA countries with a history of war and conflict areas, particularly in Afghanistan, Kuwait, Iraq, Lebanon, and more recently in Syria and Yemen. The large number of studies reporting militaryrelated TBI clearly indicates the regional constant political instabilities and perpetual wars and conflicts endured for the last decades and the adverse health implications on individuals and communities.

Fall injuries were another major cause of TBI injury in the region, particularly occupation-related injuries (i.e., falling from height at construction sites). The rapid advancement in the construction and manufacturing industry at various developing MENA countries, along with a clear absence of safety precautions and occupational safety regulations led to the dramatic increase in occupationrelated injuries. This review calls for an urgent modification of work environments and the need to implement safety measures at workplaces to reduce the number of fall-related TBI and their associated prolonged hospitalization and direct medical expenditures [74]. Falling off camels represented another aspect of fall injuries and a major cause of head traumas, particularly among children. Camels are commonly used for occupational and recreational activities in many Middle Eastern countries. Due to their small body size, children typically serve as jockeys in most camel-racing activities, hence increasing their risks of fall-related TBI. There is a need to ensure the proper use of protective gear and the implementation of safety measures to safeguard children in such risky activities. Moreover, the alarming number of reported assault- and violence-related TBIs among the pediatric population are worth highlighting. Child abuse is a significant public health problem that warrants concerted efforts to inform strict child protection policies and laws to protect children and promote their physical and mental well-being.

This review indicates that the TBI severity remarkably varied throughout the MENA countries. mTBI contributed to a significantly high incidence proportion compared to moderate and severe TBI proportions, particu- larly due to MVAs and falls; thus, agreeing with global trends of TBI-related mechanisms [75-77]. The hospitalization course and outcomes of these patients, however, are yet to be studied.

\section{Implications and Recommendations for Future \\ Research and Health Professionals}

$\mathrm{mTBI}$ nowadays is considered equally important compared to severe TBI, due to its documented long-term effects and build-up comorbidities $[1,78]$. Local physicians and neurosurgeons are urged to act more vigorously in treating $\mathrm{mTBI}$ cases and even recommending rehabilitation programs to avoid future severe physical and longterm neuropsychological disabilities. To this end, bloodbased biomarkers, increasingly being used worldwide, can represent a valuable tool to enhance the diagnostic process by enabling more accurate brain injury assessment [7981]. Moreover, their integration in clinical algorithms can improve the existing decision criteria and guide subsequent management and treatment decisions including advanced neuroimaging, hospital admission, and long-term interventions (i.e., rehabilitation programs) [82, 83].

To help address the heterogeneity of reporting, TBI databases and registries should be standardized and leveraged to include demographics such as age and gender, nationality, causes and mechanisms, and severity of TBI victims and survivors. Answers to these questions will help build a comprehensive picture of the TBI footprint for physicians, neurosurgeons, and policymakers to plan for proper, needed, and adequate intervention measures for TBI cases in the region.

In addition, there should be an initiative toward establishing biorepositories in the MENA region with standardized methodologies for collecting and reporting biospecimens from TBI cases. Such biospecimens could be assessed longitudinally for biomarker research as well as for identification and validation of the pathophysiology and etiology of TBI. The latter is of extreme importance as standardizing the reporting of the numerous and diverse TBI cases reported in the MENA region could be used for confirmatory studies in conjunction with different international TBI research centers.

Overall, this review highlighted the fact that existing literature reveals the non-standardized and non-accurate documentation of available TBI data in the MENA region, hence the demand for more standardized documentation processes, aligning with international standards to facilitate comparison and effective TBI assessment. Future studies are warranted to ensure the comparability of the epidemiological evidence base. Understanding the
28

Neuroepidemiology 2021;55:20-31 DOI: $10.1159 / 000511554$
Al-Hajj et al. 
epidemiology of TBI in the MENA region will be a fundamental step toward developing strategic TBI prevention and rehabilitation programs, allocating resources, and informing safety policies aiming at reducing brain injuries and saving lives.

\section{Limitations}

This review has some limitations. First, the heterogeneity of the included studies hindered our attempt to apply a concise meta-analytical analysis. Second, this study was unable to determine whether the samples selected in the existing studies were representative of the country's population. This sampling variation made it challenging to estimate the general TBI rate in each country and consequently in the region. Lastly, the non-standardized documentation of included TBI studies limited the accurate comparison and effective assessment of TBI prevalence, hence the demand for more standardized documentation processes in the MENA region, aligning with international standards.

\section{Conclusion}

Despite the limited TBI literature in the MENA region, this systematic review indicates that this region bears a disproportionally high prevalence of TBIs compared to global burden. The incidence and severity of TBI varied remarkably throughout the MENA countries, with mTBI contributing to a significantly high incidence proportion compared to moderate and severe TBI, mainly due to MVAs, fall-, and military-related TBI in war and conflict areas. Future studies are warranted to ensure the comparability of the TBI evidence-based studies. Leveraging of this evidence is necessary to develop programs and intervention strategies that enhance TBI diagnosis, management, and rehabilitative treatment and to mitigate TBI impacts on individuals and health-care systems in the MENA region.

\section{Acknowledgements}

We thank the Lebanese TBI working group for helping in data collection: Hisham Bahmad, Department of Anatomy, Cell Biology and Physiological sciences, Faculty of Medicine, American University of Beirut, Beirut, Lebanon. Mayse Nasser, Faculty of Medicine, Beirut Arab University, Beirut, Lebanon. Hiba Ghandour, Faculty of Medicine, American University of Beirut, Beirut,
Lebanon. Ali Kassem, Faculty of Medical sciences, Lebanese University, Beirut, Lebanon. Hadi Abou El Hassan, Faculty of Medicine, American University of Beirut, Beirut, Lebanon. Rayyan Wazzi-Mkahal, Faculty of Medicine, Beirut Arab University, Beirut, Lebanon. Lina Karout, Faculty of Medicine, Beirut Arab University, Beirut, Lebanon. Ola Assad, Faculty of Medicine, Beirut Arab University, Beirut, Lebanon. Mohamed El-Moheb, Faculty of Medicine, American University of Beirut, Beirut, Lebanon. Ahmad Chmaise, Faculty of Medicine, American University of Beirut, Beirut, Lebanon. Nour Houchiemi, Faculty of Medicine, Beirut Arab University, Beirut, Lebanon. Special thanks and great gratitude is to be offered to Miss Aida Farha, Medical Information Specialist, Saab Medical Library, American University of Beirut, Beirut, Lebanon, for her great mentoring and assistance in developing the search strategies and instructing on how to deal with the different databases.

We would also like to acknowledge the role of Hatem Mona, Razan Ghalayeini, Fatima Sultan, and Mohammad Obeid from the Medical Research Volunteer program (MRVP) for their assistance in tracking the manuscripts and assistance in screening and data extraction.

\section{Statement of Ethics}

The systematic review was conducted according to our registered protocol (PROSPERO CRD42017058952; available on: https://www.crd.york.ac.uk/prospero/display_record. php?ID=CRD42017058952). The design is consistent with the recommendations made in the "Cochrane Handbook for Systematic Reviews and Meta-Analysis," with reporting based on the Preferred Reporting Items for Systematic Reviews and Meta-Analyses (PRISMA) guidelines.

\section{Conflict of Interest Statement}

No competing financial interests exist.

\section{Author Contributions}

S.H., F.K., H.A.A., and H.T. contributed to the conception and design of the work. Z.H., J.C., M.A., M.M.M., and K.K. helped in acquisition of Data. S.M., H.P., H.H., H.T., S.H., and Z.H. conducted analysis and interpretation of data. F.K., H.A.A., H.T., and S.H. helped in drafting the article. S.H., H.T., H.P., and S.M. performed the statistical analysis. F.K. and S.H. performed study supervision. All authors have critically revised and approved the final version of the manuscript.

\section{Funding Sources}

This research was partially supported by the American University of Beirut (AUB) Medical Practice Plan (MPP) Grant: Assessment of Traumatic Brain Injury in Lebanon: A Retrospective Cohort Study; PI: Dr. Hani Tamim. 
1 Maas AIR, Menon DK, Adelson PD, Andelic $\mathrm{N}$, Bell MJ, Belli A, et al. Traumatic brain injury: integrated approaches to improve prevention, clinical care, and research. Lancet Neurol. 2017 Dec;16(12):987-1048.

2 Choi DH, Song KJ, Shin SD, Ro YS, Hong KJ, Park JH. Epidemiology and outcomes of sports-related traumatic brain injury in children. J Korean Med Sci. 2019 Nov 18;34(44): e290.

3 GBD 2017 Causes of Death Collaborators. Global, regional, and national age-sex-specific mortality for 282 causes of death in 195 countries and territories, 1980-2017: a systematic analysis for the global burden of disease study 2017. Lancet. 2018 Nov 10; 392(10159):1736-88.

4 Dewan MC, Rattani A, Gupta S, Baticulon RE, Hung YC, Punchak M, et al. Estimating the global incidence of traumatic brain injury. J Neurosurg. 2018 Apr 1:1-18.

5 El-Menyar A, Mekkodathil A, Al-Thani H, Consunji R, Latifi R. Incidence, demographics, and outcome of traumatic brain injury in the middle east: a systematic review. World Neurosurg. 2017 Nov; 107:6-21.

6 Hejase HJ, El Skaff D, Hamdar B, Hejase AJ. Trauma awareness and education in Lebanon: an exploratory study. J Middle East North Afr Sci. 2017;3(10):8-31.

7 Moher D, Liberati A, Tetzlaff J, Altman DG, Group P. Preferred reporting items for systematic reviews and meta-analyses: the PRISMA statement. BMJ. 2009 Jul 21;339:b2535.

8 Bahloul M, Chelly H, Gargouri R, Dammak H, Kallel H, Hamida CB, et al. Traumatic head injury in children in south Tunisia epidemiology, clinical manifestations and evolution. A propos 454 cases. Tunis Med. 2009;87(1):2837.

9 Grivna M, Barss P, Stanculescu C, Eid HO, Abu-Zidan FM. Child and youth traffic-related injuries: use of a trauma registry to identify priorities for prevention in the United Arab Emirates. Traffic Inj Prev. 2013;14(3): 274-82.

10 Chelly H, Bahloul M, Ammar R, Dhouib A, Mahfoudh KB, Boudawara MZ, et al. Clinical characteristics and prognosis of traumatic head injury following road traffic accidents admitted in ICU "analysis of 694 cases". Eur J Trauma Emerg Surg. 2019 Apr;45(2):245-53.

11 Abou-Raya S, ElMeguid LA. Road traffic accidents and the elderly. Geriatr Gerontol Int. 2009;9(3):290-7.

12 Eid HO, Barss P, Adam SH, Torab FC, Lunsjo $\mathrm{K}$, Grivna $\mathrm{M}$, et al. Factors affecting anatomical region of injury, severity, and mortality for road trauma in a high-income developing country: lessons for prevention. Injury. 2009; 40(7):703-7.

13 Hassan NA, Kelany RS, Emara AM, Amer M. Pattern of craniofacial injuries in patients admitted to Tanta University Hospital: Egypt. J Forensic Leg Med. 2010;17(1):26-32.
14 Al-Jazaeri A, Zamakhshary M, Al-Omair A, Al-Haddab Y, Al-Jarallah O, Al-Qahtani R. The role of seating position in determining the injury pattern among unrestrained children involved in motor vehicle collisions presenting to a level I trauma center. Ann Saudi Med. 2012;32(5):502-6.

15 Bener A. A study on road traffic crashes and injuries in Qatar as reported by drivers. J Egypt Public Health Assoc. 2012 Dec;87(5-6): 85-9.

16 Mahmood S, Parchani A, El-Menyar A, Zarour A, Al-Thani H, Latifi R. Utility of bispectral index in the management of multiple trauma patients. Surg Neurol Int. 2014; 5:141.

17 Parchani A, El-Menyar A, Al-Thani H, ElFaramawy A, Zarour A, Asim M, et al. Traumatic subarachnoid hemorrhage due to motor vehicle crash versus fall from height: a 4 -year epidemiologic study. World Neurosurg. 2014;82(5):e639-44.

18 Al-Shammari N, Bendak S, Al-Gadhi S. Indepth analysis of pedestrian crashes in Riyadh. Traffic Inj Prev. 2009;10(6):552-9.

19 Hefny AF, Eid HO, Abu-Zidan FM. Pedestrian injuries in the United Arab Emirates. Int J Inj Contr Saf Promot. 2015;22(3):203-8.

20 Hefny AF, Barss P, Eid HO, Abu-Zidan FM. Motorcycle-related injuries in the United Arab Emirates. Accid Anal Prev. 2012;49: 245-8.

21 Hefny AF, Eid HO, Grivna M, Abu-Zidan FM. Bicycle-related injuries requiring hospitalization in the United Arab Emirates. Injury. 2012;43(9):1547-50.

22 Alani M, Zarour A, Almadani A, Al-Aieb A, Hamzawi H, Maull KI. All terrain vehicle (ATV) crashes in an unregulated environment: a prospective study of 56 cases. J Emerg Med Trauma Acute Care. 2012;2012.

23 Aras M, Altaş M, Yilmaz A, Serarslan Y, Yilmaz N, Yengil E, et al. Being a neighbor to Syria: a retrospective analysis of patients brought to our clinic for cranial gunshot wounds in the Syrian civil war. Clin Neurol Neurosurg. 2014;125:222-8.

24 Karaarslan B, Uysal C, Çelikel A, Atan Y, Asildağ MK, Tataroğlu Z, et al. Evaluation of the death cases occurred as a result of injury during war. Acta Med Mediterr. 2014;30(4): 797-800.

25 Celikel A, Karbeyaz K, Kararslan B, Arslan MM, Zeren C. Childhood casualties during civil war: Syrian experience. J Forensic Leg Med. 2015 Aug;34:1-4.

26 Haddad FS. Wilder Penfield lecture: nature and management of penetrating head injuries during the civil war in Lebanon. Can J Surg. 1978 May;21(3):233-40.

27 Fosse E, Husum H, Giannou C. The siege of Tripoli 1983: war surgery in Lebanon. J Trauma. 1988 May;28(5):660-3.
28 Taha JM, Haddad FS, Brown JA. Intracranial infection after missile injuries to the brain: report of 30 cases from the Lebanese conflict. Neurosurgery. 1991 Dec;29(6):864-8.

29 Haddad FS. Penetrating missile head injuries: personal experiences during the Lebanese conflict. Neurosurg Q. 2002;12(4):299-306.

30 Fares Y, Fares J, Gebeily S. Head and facial injuries due to cluster munitions. Neurol Sci. 2014;35(6):905-10

31 McGuigan R, Spinella PC, Beekley A, Sebesta J, Perkins J, Grathwohl K, et al. Pediatric trauma: experience of a combat support hospital in Iraq. J Pediatr Surg. 2007 Jan;42(1):207-10

32 Edwards MJ, Lustik M, Eichelberger MR, Elster E, Azarow K, Coppola C. Blast injury in children: an analysis from Afghanistan and Iraq, 2002-2010. J Trauma Acute Care Surg. 2012 Nov;73(5):1278-83.

33 Behbehani A, Abu-Zidan F, Hasaniya N, Merei J. War injuries during the Gulf war: experience of a teaching hospital in Kuwait. Ann R Coll Surg Engl. 1994 Nov;76(6):407-11.

34 Marshall TJ Jr. Combat casualty care: the alpha surgical company experience during operation Iraqi freedom. Mil Med. 2005 Jun; 170(6):469-72.

35 Creamer KM, Edwards MJ, Shields $\mathrm{CH}$, Thompson MW, Yu CE, Adelman W. Pediatric wartime admissions to US military combat support hospitals in Afghanistan and Iraq: learning from the first 2,000 admissions. J Trauma. 2009 Oct;67(4):762-8.

36 Kamel MI, Youssef RM, Teleb NA, Atta HY. Epidemiology of school injuries in Alexandria. J Egypt Public Health Assoc. 1998;73(56):667-90.

37 Nawaz A, Matta H, Hamchou M, Jacobsz A, $\mathrm{Al}$ Salem AH. Camel-related injuries in the pediatric age group. J Pediatr Surg. 2005; 40(8):1248-51.

38 Kariyattil R, Rahim MI, Muthukuttiparambil U. Cerebellar mutism following closed head injury in a child. Sultan Qaboos Univ Med J. 2015;15(1):e133-5.

39 Eirale C, Farooq A, Smiley FA, Tol JL, Chalabi $\mathrm{H}$. Epidemiology of football injuries in Asia: a prospective study in Qatar. J Sci Med Sport. 2013;16(2):113-7.

40 Grivna M, Eid HO, Abu-Zidan FM. Epidemiology, morbidity and mortality from fall-related injuries in the United Arab Emirates. Scand J Trauma Resusc Emerg Med. 2014 Sep 2;22:51.

41 Dhar D. Retrospective study of injuries in military parachuting. Med J Armed Forces India. 2007 Oct; 63(4):353-5.

42 Al-Mahroos F, Al-Amer EA, Umesh NJ, Alekri AI. Pattern of skeletal injuries in physically abused children. Bahrain Med Bull. 2011; 33(2).

43 Al-Mahroos FT, Al-Amer EA, Al-Hashimi HA, Abdulla AM, Hameed N. Abusive head Trauma in children: the extent and clinical characteristics. Bahrain Med Bull. 2011;33(4): 174 
44 Al-Mahroos F, Al-Amer E. Child physical abuse in Bahrain: a 10-year study, 2000-2009. Eastern Mediterr Health J. 2012;18(6):57985.

45 Alnasser M, AlSelaim N, Aldhukair S, Elbedah K, Tamim H, Alazzam S, et al. Patterns of pediatric trauma in Ramadan: an observational study. Ann Pediatric Surg. 2012;8(1): 9-11.

46 Alsaif D, Alsowayigh K, Alfaraidy M, Albayat M, Alshamsi G, Aldosary M, et al. Child homicide in Cairo from 2006 to 2010: characteristics and trends. J Forensic Leg Med. 2013; 20(7):929-32.

47 McColl H, Higson-Smith C, Gjerding S, Omar MH, Rahman BA, Hamed M, et al. Rehabilitation of torture survivors in five countries: common themes and challenges. Int J Ment Health Syst. 2010;4:16.

48 Yuh SJ, Alaqeel A. Ten self-inflicted intracranial penetrating nail gun injuries. Neurosciences. 2015;20(3):267-70.

49 Sakr S, Azzazi A, Elsayed A, Fathalla H. Surgical management of penetrating intracranial bullet injuries. Neurosurg Q. 2016;26(1):37-41.

50 Adeloye A, Kouka N, Al-Saigh MR, Elgohary AG, Al-Manee MS. Intracranial pressure monitoring in head injury: its first use in Kuwait. Med Princ Pract. 1990;2(3-4): 156-61.

51 Jamjoom A, Jamjoom ZA, Naim-Ur-Rahman R, Tahan A, Malabarey T, Kambal A. Experience with brain abscess in the central province of Saudi Arabia. Trop Geogr Med. 1994; 46(3):154-6.

52 Asfar SN, AbdulKhadir M, Thejeel FH. The ICU outcome of head injury in Basrah, Iraq. J Neurol Orthop Med Surg. 1996;16(4):237-41.

53 Al-Harthi AS, Al-Harbi M. Accidental injuries during muslim pilgrimage. Saudi Med J. 2001 Jul;22(6):523-5.

54 Al-Naami MY, Sadik AA, Adam MA. Evaluation of trauma registry data in Asir region. Neurosciences. 2001 Jul;6(3):178-83.

55 Bahloul M, Chaari AN, Kallel H, Khabir A, Ayadi A, Charfeddine H, et al. Neurogenic pulmonary edema due to traumatic brain injury: evidence of cardiac dysfunction. Am J Crit Care. 2006 Sep;15(5):462-70.

56 Ali AM, Al-Abdulgader A, Kamal HM, AlWehedy A. Traumatic and non-traumatic coma in children in the referral hospital, AlHasa, Saudi Arabia. East Mediterr Health J. 2007;13(3):608-14.

57 Kamal H, Mardini A, Aly BM. Traumatic brain injury in pediatric age group; predictors of outcome in pediatric intensive care unit. Libyan J Med. 2007;2(2):90-4.

58 Mousali YM, Obeid TH, Al-Gahtani HA, AlJanadi MA, Attar AA, Dammas HO. Epilepsy in patients 60 years and above. Neurosciences. 2009 Jan; 14(1):53-5.

59 Arabi YM, Haddad S, Tamim HM, Al-Dawood A, Al-Qahtani S, Ferayan A, et al. Mor- tality reduction after implementing a clinical practice guidelines-based management protocol for severe traumatic brain injury. J Crit Care. 2010 Jun;25(2):190-5.

60 Shehab HA, Nassar YH. Neuromarkers as diagnostic adjuvant to cranial CT in closed traumatic brain injury patients admitted to ICU: a preliminary comparative study. Egypt J Anaesth. 2010;26(4):267-72.

61 Bouguetof H, Negadi MA, El Halimi K, Boumendil D, Mentouri ZC. Epidemiology of severe traumatic brain injury (TBI) in pediatric population of the West of Algeria. Arch Dis Child. 2012;97:A492.

62 Al-Habib A, A-Shail A, Alaqeel A, Alqeel A, Zamakhshary M, Al-Bedah K, et al. Causes and patterns of adult traumatic head injuries in Saudi Arabia: implications for injury prevention. Ann Saudi Med. 2013 Jul-Aug;33(4): 351-5.

63 Imen R. B, Olfa C, Kamilia C, Meriam B, Hichem K, Adel C, et al. Factors predicting early outcome in patients admitted at emergency department with severe head trauma. J Acute Dis. 2015;4(1):68-72.

64 Boudokhane SS, Migaou H, Salah S, Jellad A, Ben Salah Frih Z. Outcome one year post severe traumatic brain injury: about a Tunisian population. Ann Phys Rehabil Med. 2015; 58(1):14-5

65 Al-Adawi S, Al-Naamani A, Al-Farsi Y, Essa M, Burke D, El-Bouri M, et al. Elucidating the neuropsychological profile of apathetic syndrome and disinhibition syndrome in a braininjured population in Oman. Int J Nutr Pharmacol Neurol Dis. 2016;6(1):35-45.

66 Bener A, Al-Mulla FH, Al-Humoud SM, Azhar A. Camel racing injuries among children. Clin J Sport Med. 2005;15(5):290-3.

67 Ralli G, Giovanni R, Atturo F, Francesca A, Lamberti A, Antonio L, et al. Post-traumatic camel-related benign paroxysmal positional vertigo. Travel Med Infect Dis. 2010 Jul;8(4): 207-9.

68 Al-Ali MA, Hefny AF, Abu-Zidan FM. Head, face and neck camel-related injuries: biomechanics and severity. Injury. 2019 Jan;50(1): 210-4.

69 Droussi H, Amrani DE, Dlimi M, Elatiqi K, Boukind S, Exadaktylos AK, et al. Domestic donkey (Equus africanus asinus) bites: an unusual aetiology of severe scalp injuries in Morocco. Afr J Emerg Med. 2015;4(4):e5-8.

$70 \mathrm{Hoz}$ SS, Dolachee AA, Abdali HA, Kasuya H. An enemy hides in the ceiling; pediatric traumatic brain injury caused by metallic ceiling fan: case series and literature review. Br J Neurosurg. 2019 Jun;33(3):360-4.

71 Tagliaferri F, Compagnone C, Korsic M, Servadei F, Kraus J. A systematic review of brain injury epidemiology in Europe. Acta Neurochir. 2006 Mar;148(3):255-68; discussion 68

72 Nguyen R, Fiest KM, McChesney J, Kwon CS, Jette N, Frolkis $A D$, et al. The international incidence of traumatic brain injury: a systematic review and meta-analysis. Can J Neurol Sci. 2016 Nov;43(6):774-85.

73 Al-Hajj S, El Bcheraoui C, Daoud F, Khalil I, Moradi-Lakeh M, Abu-Raddad LJ, et al. Child and adolescent injury burden in the Eastern Mediterranean region: findings from the global burden of disease 1990-2017. BMC Public Health. 2020 Apr 3;20(1):433.

74 Davoodi HR, Abed GT. Challenges of growth and globalization in the middle east and north Africa. Washington, DC: International Monetary Fund; 2003.

75 Asim M, El-Menyar A, Al-Thani H, Abdelrahman $\mathrm{H}$, Zarour A, Latifi R. Blunt traumatic injury in the Arab Middle Eastern populations. J Emerg Trauma Shock. 2014 Apr;7(2): 88-96.

76 Brazinova A, Rehorcikova V, Taylor MS, Buckova V, Majdan M, Psota M, et al. Epidemiology of traumatic brain injury in Europe: a living systematic review. J Neurotrauma. 2018 [Online ahead of print].

77 Capizzi A, Woo J, Verduzco-Gutierrez M. Traumatic brain injury: an overview of epidemiology, pathophysiology, and medical management. Med Clin North Am. 2020 Mar; 104(2):213-38.

78 Barnes DE, Byers AL, Gardner RC, Seal KH, Boscardin WJ, Yaffe K. Association of mild traumatic brain injury with and without loss of consciousness with dementia in US military veterans. JAMA Neurol. 2018 Sep 1; 75(9):1055-61.

79 Mondello S, Muller U, Jeromin A, Streeter J, Hayes RL, Wang KK. Blood-based diagnostics of traumatic brain injuries. Expert Rev Mol Diagn. 2011 Jan;11(1):65-78.

80 Mondello S, Sorinola A, Czeiter E, Vamos Z, Amrein K, Synnot A, et al. Blood-based protein biomarkers for the management of traumatic brain injuries in adults presenting to emergency departments with mild brain injury: a living systematic review and metaanalysis. J Neurotrauma. 2018 [Online ahead of print]

81 Czeiter E, Amrein K, Gravesteijn BY, Lecky F, Menon DK, Mondello S, et al. Blood biomarkers on admission in acute traumatic brain injury: relations to severity, CT findings and care path in the CENTER-TBI study. EBioMedicine. 2020 May 25;56:102785.

82 Kou Z, Gattu R, Kobeissy F, Welch RD, O'Neil BJ, Woodard JL, et al. Combining biochemical and imaging markers to improve diagnosis and characterization of mild traumatic brain injury in the acute setting: results from a pilot study. PLoS One. 2013;8(11):e80296.

83 Mondello S, Schmid K, Berger RP, Kobeissy F, Italiano D, Jeromin A, et al. The challenge of mild traumatic brain injury: role of biochemical markers in diagnosis of brain damage. Med Res Rev. 2014 May;34(3): 503-31. 